\title{
Vehicle routing: historical perspective and recent contributions
}

\author{
Gilbert Laporte $\cdot$ Paolo Toth $\cdot$ Daniele Vigo
}

Received: 9 January 2013/Accepted: 17 January 2013/Published online: 15 March 2013

(c) Springer-Verlag Berlin Heidelberg and EURO - The Association of European Operational Research Societies 2013

\begin{abstract}
The vehicle routing problem requires the design of least cost delivery routes through a set of geographically scattered customers and is one of the most widely studied combinatorial optimization problems. We briefly summarize the main historical achievements and review some recent important contributions in this vivid research area.
\end{abstract}

\section{Historical Perspective}

The vehicle routing problem (VRP) consists of designing least cost delivery routes through a set of geographically scattered customers, subject to a number of side constraints. This problem holds a central place in distribution management and is faced on a daily basis by tens of thousands of carriers worldwide. The problem arises in several forms because of the variety of constraints encountered in practice. For over 50 years, the VRP has attracted the attention of a large part of the operational research community. This is due partly to the economic importance of the problem, but also to the methodological challenges it poses. For example, the traveling salesman problem (TSP), which is a special case of the VRP, can now be solved for thousands and even tens of thousands of vertices. In contrast, the VRP is much more difficult to solve. For example, in the relatively simple case where only

The EURO Journal on Transportation and Logistics (EJTL) has chosen Vehicle Routing as the theme of one of its first special issues. The three guest editors are very pleased to be part of this initiative.

G. Laporte

HEC Montréal, Montréal, Canada

P. Toth · D. Vigo $(\bowtie)$

DEI, Alma Mater Studiorum Università di Bologna, Bologna, Italy

e-mail: daniele.vigo@unibo.it 
capacity constraints are present (called the capacitated VRP, CVRP), it is still difficult to solve instances with one or two hundred customers by means of exact algorithms. In recent years, much of the research effort have turned to the development of powerful metaheuristics.

The capacitated VRP was formally introduced in 1959 by Dantzig and Ramser (1959). These authors proposed a simple matching-based heuristic for its solution and illustrated it on a toy-sized example. The following years saw the emergence of several heuristics based on a variety of principles including savings, geographical proximity, customer matchings, as well as intra-route and inter-route improvement steps. Perhaps the most famous heuristic of this category is the Clarke and Wright (1964) savings heuristic, which has withstood the test of time because of its speed, simplicity and reasonably good accuracy. The development of exact algorithms for the VRP took off in 1981 with the publication of two papers by Christofides, Mingozzi and Toth in Networks, Christofides et al. (1981) and Mathematical Programming, Christofides et al. (1981). The first of these papers proposed an algorithm based on dynamic programming with state-space relaxation whereas the second proposed two mathematical formulations making use of $q$-paths and $k$-shortest spanning trees. A few years later, Laporte, Desrochers and Nobert, Laporte et al. (1984) proposed the first cutting plane approach for a VRP based on the solution of linear relaxation of an integer model. These seminal concepts have made their way into some of the more recent algorithms.

Since then, a variety of exact algorithms based on mathematical programming formulations have been proposed. Some formulations contain vehicle flow or commodity flow variables and are often solved by branch-and-cut. The VRP can also be formulated as a set partitioning problem to which some valid inequalities are added. Some of the most successful implementations by Fukasawa et al. (2006) and by Baldacci et al. (2008), are based on this methodology.

The development of modern heuristics for the VRP really started in the 1990s with the advent of metaheuristics. It is fair to say that the study of the VRP has stimulated the growth and understanding of several metaheuristic concepts we now know. The early research in this area was quite fragmented, with a notable bias towards tabu search-based approaches [see Taillard (1993), Gendreau et al. (1994), and Toth and Vigo (2003)] and some of the algorithms were over engineered, but some rationalization has started to take place in recent years. The best metaheuristics are those that simultaneously perform a wide and deep search of the solution space and can solve several variants of the problem. They generally either apply several operators, as in adaptive large neighbourhood search (Pisinger and Ropke 2007), or combine genetic search with local search, as in the hybrid genetic algorithm recently proposed by Vidal et al. (2012).

\section{Recent contributions}

This special issue of EJTL contains seven papers. The first, by Abercrombie Lindsey, Erera and Savelsbergh, describes a pickup and delivery problem in which freight is moved from suppliers to distribution centers and shipment consolidation 
may take place at crossdock facilities. Small instances can be solved exactly, and a search scheme was developed for the solution of larger instances. It was tested on data from a major US retailer. The second paper, by Cappanera, Gouveia and Scutellà, introduces models and valid inequalities for a variety of problems involving the routing of technicians with specialized skills. Such applications arise in the area of field service and of home care delivery. The third paper, by Stenger, Schneider and Goeke, deals with the prize-collecting problem with one and several depots and non-linear costs. This problem arises in the area of small package shipping. The authors propose an adaptive variable neighbourhood search heuristic for it. The fourth paper, by Voccia, Campbell and Thomas, considers a probabilistic version of the TSP with time windows in which the presence of customers is probabilistic. The authors present a recourse model as well as a variable neighbourhood descent algorithm for its solution.

The fifth paper, by Quttineh, Larsson, Lundberg and Holmberg, introduces a military aircraft mission planning problem in which a fleet of aircraft must attack a number of ground targets. The problem is cast as a generalized VRP with synchronization and precedence constraints. It is formulated as a linear mixed integer program and solved by CPLEX for instances of realistic sizes.

The last two papers of this special issue, by Brodtkorb, Hagen, Schulz and Hasle, present a survey on the use of GPU (Graphics Processing Unit) in discrete optimization and in routing problems. The focus of these papers is on GPU-based methods which make use of parallel processors assigned to different pieces of distributed data. The use of these systems is central to the solution of large applications in which huge data sets must be processed, often in real-time.

Acknowledgments We thank the authors for their contributions and the referees for their thorough and timely reviews. We are proud to present a rich assortment of high quality papers to the transportation and logistics research community.

\section{References}

Baldacci R, Christofides N, Mingozzi A (2008) An exact algorithm for the vehicle routing problem based on the set partitioning formulation with additional cuts. Math Program Ser A 115:351-385

Christofides N, Mingozzi A, Toth P (1981) Exact algorithms for the vehicle routing problem based on the spanning tree and shortest path relaxations. Math Program 20:255-282

Christofides N, Mingozzi A, Toth P (1981) State-space relaxation procedures for the computation of bounds to routing problems. Networks 11:145-164

Clarke G, Wright JV (1964) Scheduling of vehicles from a central depot to a number of delivery points. Oper Res 12:568-581

Dantzig GB, Ramser JH (1959) The truck dispatching problem. Manag Sci 6:80

Fukasawa R, Longo H, Lysgaard J, Poggi de Aragáo M, Reis M, Uchoa E, Werneck RF (2006) Robust branch-and-cut-and-price for the capacitated vehicle routing problem. Math Program Ser. A 106:491-511

Gendreau M, Hertz A, Laporte G (1994) A tabu search heuristic for the vehicle routing problem. Manag Sci 40:1276-1290

Laporte G, Desrochers M, Nobert Y (1984) Two exact algorithms for the distance-constrained vehicle routing problem. Networks 14:161-172

Pisinger D, Ropke S (2007) A general heuristic for vehicle routing problems. Comput Oper Res 34:2403-2435

Taillard ED (1993) Parallel iterative search methods for vehicle routing problems. Networks 23:661-673 
Toth P, Vigo D (2003) The granular tabu search and its application to the vehicle-routing problem. INFORMS J Comput 15:333-346

Vidal T, Crainic TG, Gendreau M, Prins C (2012) A unified solution framework for multi-attribute vehicle routing problems. Tech Rep, CIRRELT 